\title{
La pathologie des insectes au service du génie génétique
}

Les premières productions de protéines humaines d'intérêt biologique ou thérapeutique par génie génétique ont fait appel à des vecteurs d'expression dans lesquels le gène à exprimer est inséré. Cette construction est intégrée dans une cellule bactérienne hôte, dont on utilise la machinerie de synthèse de protéines. A l'usage, cette ère de "la bactérie au service de l'homme " a cependant révélé ses limites :

- La protéine étrangère peut être dégradée dans la bactérie, obligeant à des moyens détournés de protection (protéines de fusion, bactéries mutantes...).

- La protéine synthétisée reste le plus souvent séquestrée dans des inclusions, nécessitant une extraction dénaturante et une renaturation laborieuse dans le but de lui restituer une structure tertiaire fonctionnelle.

- Les cellules procaryotes ne réalisent pas les étapes de maturation des protéines fonctionnant chez les eucaryotes (coupures sélectives, hydroxylations, glycosylations, phosphorylations...).

- Les processus d'assemblage posttraductionnel des polypeptides synthétisés dans les cellules eucaryotes mettent en jeu une machinerie complexe impliquant des protéines spécifiques (chaperons) qui participent au transfert transmembranaire et au guidage des réactions nécessaires au repliement et au compactage corrects des chaînes peptidiques (ponts disulfures, isomérisation des prolines...).

Pour toutes ces raisons, l'utilisation de cellules eucaryotes comme hôte d'expression génétique apparaît souhaitable. Cependant, pour être maintenues en culture de masse, ces cellules doivent être "immortali$\mathrm{m} / \mathrm{s} n^{\circ} 3$ vol. 6 , mars 90

\begin{tabular}{|c|c|c|c|c|}
\hline \multicolumn{5}{|c|}{$\begin{array}{c}\text { Tableau I } \\
\text { QUELQUES PROTÉINES RECOMBINANTES OBTENUES DANS } \\
\text { LE SYSTÉME DE CELLULES D'INSECTES INFECTÉES PAR LE BACULOVIRUS }\end{array}$} \\
\hline Gène & $\begin{array}{l}\text { Protéine } \\
\text { (PM) } \\
\text { (KDa) }\end{array}$ & $\begin{array}{l}\text { Production } \\
\text { par litre de } \\
\text { cellules SF9 }\end{array}$ & $\begin{array}{l}\text { Propriétés } \\
\text { biologiques }\end{array}$ & ences \\
\hline $\begin{array}{l}\text { AcNPV } \\
\text { polyhédrine }\end{array}$ & 29 & $1000 \mathrm{mg}$ & $\begin{array}{l}\text { phosphorylée } \\
\text { localisation nucléaire }\end{array}$ & [2] \\
\hline $\begin{array}{l}c-m y c \\
\text { humain }\end{array}$ & $64 / 61$ & $1 \mathrm{mg}$ & $\begin{array}{l}\text { antigénique, } \\
\text { phosphorylée } \\
\text { localisation nucléaire }\end{array}$ & [6] \\
\hline $\begin{array}{l}\text { Interféron } \alpha \\
\text { humain }\end{array}$ & 19,5 & $0,3 \mathrm{mg}$ & $\begin{array}{l}\text { activité interféron } \alpha \\
\text { antigénique, peptide } \\
\text { signal clivé, sécrété }\end{array}$ & [7] \\
\hline $\begin{array}{l}\text { Interféron } \beta \\
\text { humain }\end{array}$ & $17 / 20,5$ & - & $\begin{array}{l}\text { activité interféron } \beta \text {, } \\
\text { antigénique, glycosylé, } \\
\text { peptide signal clivé, } \\
\text { sécrété }\end{array}$ & [8] \\
\hline $\begin{array}{l}\text { Enveloppe } \\
\text { du HIV }\end{array}$ & $150 / 120$ & - & $\begin{array}{l}\text { antigénique glycosylée, } \\
\text { protéolysée }\end{array}$ & [9] \\
\hline $\begin{array}{l}\text { Interleukine-2 } \\
\text { humaine }\end{array}$ & $16 / 15,5$ & $1 \mathrm{mg}$ & $\begin{array}{l}\text { activité interleukine-2 } \\
\text { antigénique, peptide } \\
\text { signal clivé, sécrété }\end{array}$ & [10] \\
\hline $\begin{array}{l}\text { Domaine } \\
\text { kinase } \\
\text { du récepteur } \\
\text { de l'EGF }\end{array}$ & 66 & $\begin{array}{c}2 \mathrm{~d} \\
2,5 \mathrm{mg}\end{array}$ & $\begin{array}{l}\text { activité tyrosine kinase, } \\
\text { antigénique }\end{array}$ & [11] \\
\hline $\begin{array}{l}\text { lsoforme } \gamma \text { de } \\
\text { la protéine } \\
\text { kinase C }\end{array}$ & $82-84$ & $\begin{array}{c}5 \mathrm{a} \\
15 \mathrm{mg}\end{array}$ & $\begin{array}{l}\text { activité kinase, } \\
\text { antigénique, soluble }\end{array}$ & [12] \\
\hline Ha-ras-p21 & 21 & $20 \mathrm{mg}$ & $\begin{array}{l}\text { activité antigénique, } \\
\text { membranaire, palmitoylée }\end{array}$ & [13] \\
\hline $\begin{array}{l}\text { Domaine } \\
\text { cytoplasmique } \\
\text { du récepteur } \\
\text { de l'insuline } \\
\text { humaine }\end{array}$ & 48 & $4 \mathrm{mg}$ & $\begin{array}{l}\text { activité kinase soluble, } \\
\text { antigénique }\end{array}$ & [14] \\
\hline
\end{tabular}


sées ", par exemple par des virus. Les produits émanant de telles cellules posent des problèmes quant à leur garantie d'inocuité et leur acceptation comme agent thérapeutique.

Dans ce contexte, la production de protéines recombinantes dans des cellules eucaryotes d'insectes, sous le contrôle d'un virus spécifique (baculovirus) constitue un nouveau système dont les avantages devraient entraîner une large utilisation [1]. Il permet la production abondante de protéines recombinantes, procaryotes ou eucaryotes, qui possèdent des propriétés antigéniques, immunologiques et fonctionnelles identiques à celles des protéines naturelles (Tableau I). De plus, les baculovirus ne sont pathogènes ni pour les vertébrés, ni pour les plantes.

Autographa californica nuclear polyhedrosis virus (AcNPV) [2] est le virus prototype de la famille des baculovirus qui peuvent infecter plus de 30 espèces d'insectes lépidoptères (figure 1). Au cours de l'infection, deux formes de virus sont produites par les cellules cibles : des particules virales extracellulaires (PVE) et des occlusions virales (OV) (figure 1). Ces dernières (polyhèdres), sont constituées de la particule virale entourée d'une coque protéique formée en majorité de polyhédrine. Dans les cultures de cellules d'insecte infectées, la polyhédrine (PM $29 \mathrm{kDa}$ ) s'accumule jusqu'à des concentrations de l'ordre de $1 \mathrm{mg} / \mathrm{ml}$ pour $10^{6}$ cellules infectées, ce qui représente alors plus de $50 \%$ des protéines totales de la cellule.

Le gène de la polyhédrine n'est pas essentiel pour la réplication ou la production de particules virales extracellulaires [3]. Il est dès lors possible de le modifier par l'insertion de gènes étrangers. Cette insertion, entre les régions régulatrices du gène de la polyhédrine, conduit à la production de virus sans occlusion $\left(\mathrm{OCC}^{-}\right)$qui forment des plages de lyse visuellement très différentes de celles du type sauvage à occlusion $\left(\mathrm{OCC}^{+}\right)$. Cette différence d'aspect des plages de lyse constitue un moyen visuel simple pour trier et iso-
Ces virus recombinants sont obtenus en trois étapes (figure 2) [4, 5].

1. Insertion du gène à exprimer dans un vecteur de transfert bactérien (pUC8). L'insertion se fait au niveau d'un site de restriction entouré de séquences virales encadrant le promoteur de la polyhédrine. 2. L'ADN du baculovirus et le vecteur de transfert sont cotransfectés dans les cellules d'insectes SF9 (cellules ovariennes provenant de Spodoptera frugiperda) par la technique au phosphate de calcium. Au cours de cette étape, une recombinaison homologue permet la production d'un $\mathrm{ADN}$ viral recombinant porteur du gène à exprimer.

3. Cette recombinaison est un événement rare et il est nécessaire de purifier le virus recombinant. Cela se fait par infections successives des cel- lules SF9 et identification visuelle des plages de lyse $\mathrm{OCC}^{-}$. Généralement, après trois ou quatre cycles d'enrichissement, le virus sécrété est pur, prêt à être utilisé pour infecter en masse des cellules SF9.

L'utilisation de ce système d'expression a déjà permis d'obtenir un certain nombre de protéines recombinantes eucaryotes (Tableau I). Le facteur de croissance interleukine 2 et l'interféron $\alpha$ et $\beta[7,8,10]$ sont synthétisés et sécrétés sous une forme fonctionnellement active ; la production du domaine catalytique du récepteur du facteur de croissance EGF [11] et de la protéine kinase C [12] a permis l'obtention de ces deux protéines kinases pleinement fonctionnelles.

Un intérêt supplémentaire de cette technique est le relatif faible coût des

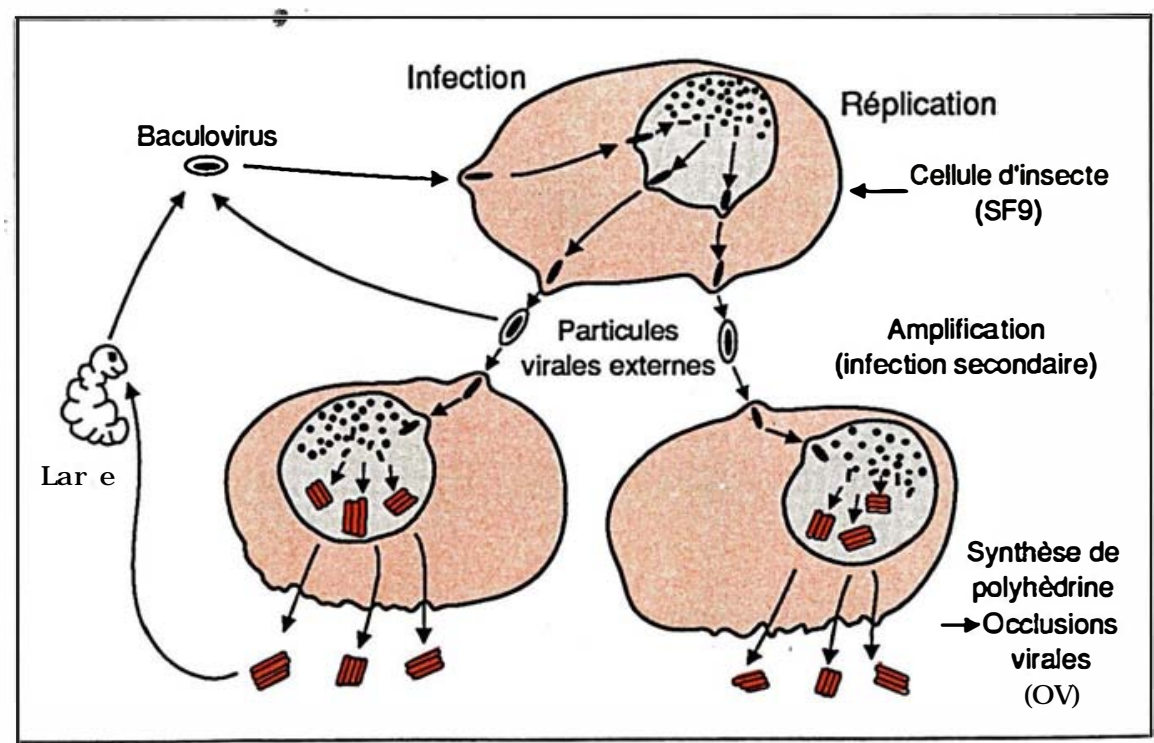

Figure 1. Dans l'environnement naturel, une larve d'insecte sensible au baculovirus est susceptible d'ingérer des occlusions virales contenues dans sa nourriture. Les cristaux de polyhédrine constituant ces occlusions se dissolvent dans l'intestin du lépidoptère, libérant les particules virales infectieuses qui envahissent alors les cellules intestinales. Une fois dans les noyaux cellulaires, l'ADN viral commence à se répliquer dès six heures après l'infection. Entre 10 et $12 \mathrm{~h}$, les premières particules virales extracellulaires quittent la cellule pour infecter les cellules voisines. Plus tard (18-24 h), la synthèse énorme de polyhédrine permet l'insertion des particules virales dans des occlusions protéiques. Ces occlusions, en s'accumulant dans la cellule jusqu'à sa lyse (3-4 jours), jouent un rôle important dans le cycle naturel du virus : quand les larves infectées meurent, des millions de cristaux de polyhédrine sont libérés des tissus en décomposition. 


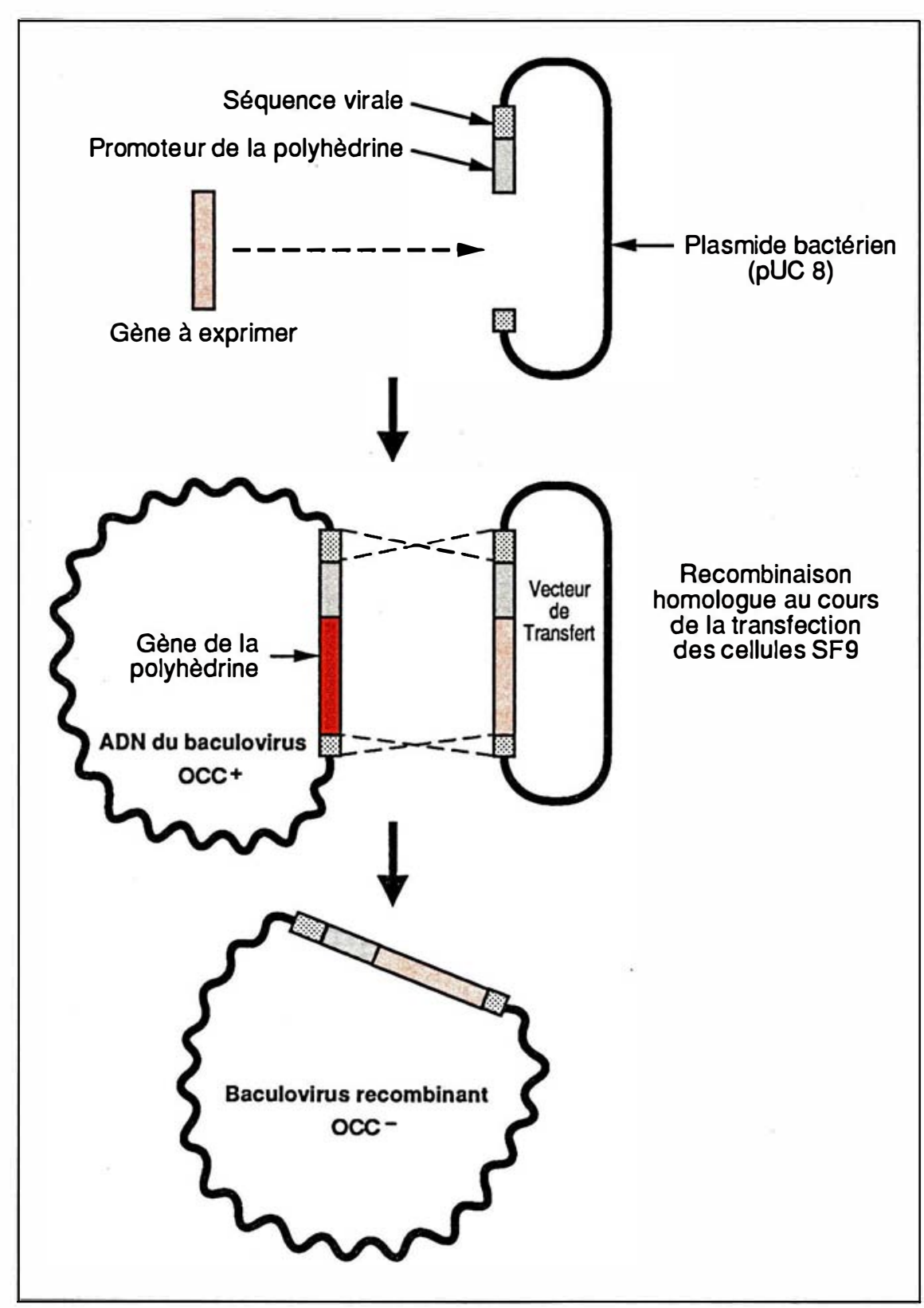

Figure 2. Schéma représentant le processus de clonage d'un gène à exprimer dans I'ADN viral. Un fragment d'ADN approprié du gène est inséré dans un vecteur de transfert dans la bonne orientation en aval du promoteur de la polyhédrine. Des cellules d'insecte (SF9) en culture sont transfectées avec un mélange d'ADN viral et de vecteur de transfert. Au cours de cette transfection, une double recombinaison homologue se produit, entraînant la formation d'ADN viral dans lequel le gène de structure de la polyhédrine a été remplacé par le gène à exprimer.

$\mathrm{m} / \mathrm{s} n^{\circ} 3$ vol. 6 , mars 90 milieux de culture récemment développés qui, sans adjonction de sérum, permettent la prolifération des cellules d'insectes, la réplication des virus et l'expression des protéines recombinantes de façon satisfaisante. Enfin, les cellules SF9 utilisées prolifèrent en suspension, permettant leur culture en fermenteur et une application à une échelle industrielle [5].

Les recherches en cours visent à optimiser le lieu d'insertion du gène à exprimer dans le vecteur de transfert en vue d'augmenter le taux d'expression de la protéine [4]. On peut imaginer qu'à terme, une manipulation directe de l'ADN du baculovirus $(\approx 130 \mathrm{~kb}$ ) permettra d'éviter l'étape de recombinaison réciproque actuellement utilisée pour la construction du vecteur. La méthode est appliquée dans notre laboratoire en vue d'obtenir une protéine kinase eucaryote oligomérique fonctionnelle : la caséine kinase II (CKG), formée de deux sous-unités différentes, de stoéchiométrie $\alpha_{2} \beta_{2}$ [15]

Odile Filhol

Claude Cochet

Edmond M. Chambaz

Inserm U.244, BRCE/LBIO/DRF, centre d'études nucléaires, $85 X, 38041 \mathrm{Gre}$ noble Cedex, France.

\section{RÉFÉRENCES}

1. Summers MD, Smith GE. A manual of methods for baculovirus vectors and insect cell culture procedures. Texas Agricultural Experiment Station, Bull $\mathrm{n}^{\circ}$ 1555, 1987.

2. Maruniak JE, Summers MD. Autographa californica nuclear polyhedrosis virus phosphoproteins and synthesis of intracellular proteins after virus infection. Virology 1981 ; 109 : 25-34

3. Smith GE, Fraser MJ, Summers MD. Molecular engineering of Autographa californica nuclear polyhedrosis virus genome : deletion mutations within the polyhedrin gene. J Virol 1983 ; 46 : 584-93. 


\section{RÉFÉRENCES (suite)}

4. Miller DW, Safer P, Miller LK. An insect baculovirus host-vector system for high-level expression of foreign genes. In : Setlow JK, Hollander A, eds. Genetic engineering. Principles and Methods, New York Plenum Publ Corp, 1986 ; 8 : 277-98.

5. Inlow D, Harano D, Maiorella B Large-scale insect culture for recombinan protein production. Presented at symposiurn on strategies in cell-culture scale up. New Orleans: American Chemical Society, 1987.

6. Miyamoto C, Smith GE, Farrell-Towt J, Chizzonite R, Summers MD, Ju G. Production of human c-myc protein in insect cells with a baculovirus expression vector. Mol Cell Biol 1985 ; 5 : 2860-5.

7. Maeda S, Kawai $\mathrm{T}$, Obinata M, et al. Production of human $\alpha$ interferon in silkworm using a baculovirus vector. Nature $1985 ; 315: 592-4$

8. Smith GE, Summers MD, Fraser MJ Production of human $\beta$ interferon in insect cells infected with a baculovirus expression vector. Mol Cell Biol 1983 ; 3 : 2156-65.

9. Hu SL, Kosowski SG, Schaaf KF Expression of envelope glycoproteins of human immunodeficiency virus by an insect virus vector. $J$ Virol $1987 ; 61: 820-32$

10. Smith GE, Ju G, Ericson BL, el al. Modification and secretion of human interleukine- 2 produced in insect cells by a baculovirus expression vector. Proc Natl dcad Sci USA 1985; 82 : 8404-8.

11. Wedegaertner PB, Gill GN. Activation of the purified protein tyrosine kinase domain of the epidermal growth factor receptor. J Biol Chem 1989 ; 264: 11346-53.

12. Patel G, Stabel S. Expression of a func tional protein kinase $\mathrm{C} \gamma$ using a baculovirus vector : purification and characterization of a simple protein kinase C isoenzyme. Cell Signal $1989 ; 1: 227-40$

13. Page MJ, Hall A, Rhodes $\mathrm{S}$, el al. Expression and characterization of (Ha-ras p21) protein produced at high levels in insect/baculovirus system. J Biol Chem 1989 ; $264: 19147-54$

14. Herrera R Lebwohl D, de Herreros AG, Kallen RG, Rosen OM. Synthesis, purification and characterization of the cytoplasmic domain of the human insulin receptor using a baculovirus expression system. $J$ Biol Chem $1988 ; 263$ : 5560-8.

15. Cochet C, Chambaz EM. Oligomeric structure and catalytic activity of $G$ type casein kinase. J Biol Chem 1983; 258: 1403-6. 